\title{
AVANT-PROPOS / FOREWORD
}

\section{Évaluation par les pairs (peer reviewing)}

(C) Springer-Verlag France 2010

L'évaluation par les pairs est un principe fondamental de la publication de travaux dans des revues scientifiques. Elle assure la pertinence et la validité des résultats décrits qui, afin d'être acceptés, doivent d'abord faire l'objet d'une critique attentive par un nombre restreint d'experts nommés par le comité éditorial de la revue. Le Journal africain d'hépatogastroentérologie $(J A H G)$ met à contribution deux experts en moyenne par article évalué.

Ces évaluateurs sont des spécialistes du sujet du manuscrit examiné qui acceptent bénévolement de le lire, de le commenter et parfois de le réviser en profondeur. Leur mérite est d'autant plus grand que leur nom n'apparaît nulle part dans la revue, ni associé à l'article qu'ils ont évalué, ni dans la lettre accompagnant leurs commentaires à l'auteur. L'anonymat de leur prestation fait partie de leur mission.

Nous avons décidé, ici, de les remercier pour leur soutien altruiste. Les personnes qui sont citées ci-dessous — par ordre alphabétique — ont toutes contribué à l'évaluation de plus de 150 articles soumis au JAHG en 2009.

$\begin{array}{lll}\text { Abramowitz L. } & \text { Essamri W. } & \text { Luyasu V. } \\ \text { Aubry P. } & \text { Fall F. } & \text { Mainguet P. } \\ \text { Bastid C. } & \text { Farthouat P. } & \text { Mion F. } \\ \text { Baton O. } & \text { Fiasse R. } & \text { Mollard R. } \\ \text { Bertrand C. } & \text { Florent C. } & \text { Najjar } \\ \text { Bossali F. } & \text { Garcia J.-F. } & \text { Ndiaye B. } \\ \text { Bouchaud O. } & \text { Grimaud J.-C. } & \text { Ndiaye F. } \\ \text { Bourlière M. } & \text { Hagège H. } & \text { Nouel O. } \\ \text { Brenard R. } & \text { Henrion J. } & \text { Ntagirabiri R. } \\ \text { Buset M. } & \text { Heyres L. } & \text { Rey P. } \\ \text { Cadiot G. } & \text { Houlgatte A. } & \text { Robaszkiewicz M. } \\ \text { Cador L. } & \text { Ibrahimi A. } & \text { Ruszniewski P. } \\ \text { Calès P. } & \text { Imbert P. } & \text { Sarre S. } \\ \text { Calvary R. } & \text { Kanouni N. } & \text { Sastre B. } \\ \text { Camara B. } & \text { Klotz F. } & \text { Sawadogo A. } \\ \text { Camoi T. } & \text { Lamy V. } & \text { Sassenou I. } \\ \text { Capdevielle P. } & \text { Landi B. } & \text { Siproudhis L. } \\ \text { Couzigou P. } & \text { Larrey D. } & \text { Sombie R. } \\ \text { De Maeght S. } & \text { Laterre E. } & \text { Stainier L. } \\ \text { Debongnie J.-C. } & \text { Laugier R. } & \text { Tarrieraz A.L. } \\ \text { Debzi N. } & \text { Lemoine O. } & \text { Tzeuton C. } \\ \text { Draguet A. } & \text { Letard J.-C. } & \text { Zarski J.-P. } \\ \text { Dugernier T. } & \text { Letoublon C. } & \\ \text { Dumurgier C. } & \text { Levy P. } & \\ & & \end{array}$

